\title{
LA RETÓRICA ARISTOTÉLICA Y LA ORATORIA DE SU TIEMPO (SOBRE EL EJEMPLO DE LISIAS III) ${ }^{1}$
}

\author{
FranCISCO CORTÉs GABAUDAN \\ Universidad de Salamanca
}

Aristotle's Rhetoric is a descriptive text to a greater extent than normally recognized and so our understanding of Aristotle can be improved with the analysis of speeches previous to him; as a case study, the third speech of Lysias has been choosen. The argumentation of judicial speeches previous to Aristotle was built with enthymemes whose first premises are based on emotions and characters explaining human behaviour; therefore the first purpose of Rhetoric II.2-11 (about emotions) and II.2-17 (about characters) was to supply the orators with first premises. In those speeches the emotions of the audience were stired up by enthymemes; therefore Aristotle accepted that the orator could use emotions in this rational and logic way. Nevertheless he condemned the arousing of emotions by direct and irrational requests, a procedure found in the judicial speeches. This way the apparent contradictory treatment of emotions in Aristotle's Rhetoric can be solved.

\section{Retórica y oratoria}

La Retórica ${ }^{2}$ de los siglos V y IV a.C., anterior a Aristóteles, tenía como finalidad fundamental facilitar la composición de discursos, especialmente del género judicial ${ }^{3}$. Era práctica y funcionaba de forma descriptiva: se nutría de los discursos, hacía un repertorio de sus elementos,

1 Este trabajo se ha realizado en el marco del Proyecto de Investigación DGICYT, PB 96-1268.

2 Bastaría citar a Navarre o el trabajo de Lavency. Ejemplos prácticos de este proceder pueden verse en nuestros trabajos de 1986 y 1989.

3 Sobre la preeminencia del género judicial, véase Hellwig, pp. 155 ss. y nuestro trabajo de 1994 a. Cf. Cole, pp. 71 ss. 
recursos compositivos, etc., tomaba de ellos elementos intercambiables, reaprovechables que agrupaba probablemente en referencia a las distintas partes del discurso para facilitar su reutilización. Su planteamiento práctico y poco teórico suponía una escasa profundización en los procedimientos persuasivos o argumentativos, aunque desde el punto de vista de Platón o Aristóteles, su gran hallazgo fuera el del razonamiento por lo verosímil ( $\varepsilon i$ Kós). Probablemente algunos sofistas fueron más allá y prepararon el terreno a las críticas de esta concepción empobrecedora de la Retórica que se formularon en el s. IV, por parte de Platón, Isócrates y Aristóteles ${ }^{4}$.

Platón, como es bien sabido, criticó profundamente esta situación con la intención clara de modificar la teoría retórica y la práctica de los discursos, por tanto, su perspectiva era claramente normativa, en el sentido de que intentaba modificar la realidad existente, tanto en la esfera de la oratoria, como en la de la Retórica. Nos vamos a fijar especialmente en las críticas platónicas ${ }^{5}$ del Fedro que más incidieron en el desarrollo retórico posterior:

a) los rétores prefieren argumentar por lo verosímil ( $\varepsilon i$ kós) en lugar de hacerlo según la verdad ${ }^{6}$.

b) la Retórica como $\psi v \chi \alpha \gamma \omega \gamma$ ía debería proporcionar un profundo conocimiento previo del alma del interlocutor para poder convencerlo ${ }^{7}$.

c) el discurso debe ser coherente, integrado ${ }^{8}$.

d) la Retórica se ha dedicado a temas periféricos, como las partes del discurso, y además se ha restringido al terreno de la oratoria judicial, crítica ésta última en la que coincidía plenamente Isócrates ${ }^{9}$.

Aristóteles se esforzó en elaborar una Retórica que diese respuestas a las críticas platónicas, intentando al tiempo conservar muchos de los elementos de la retórica y oratoria anteriores. La postura aristotélica es un

\footnotetext{
Sobre las críticas de Platón e Isócrates puede verse el reciente trabajo de Laplace.

5 Véase, p. ej., Kroll.

P1., Phaedr. 272d-e.

P1., Phaedr. 271c-d.

8 P1., Phaedr. 264c.

9 P1., Phaedr. 261b y 266d-267a.
} 
compromiso entre las propuestas normativas platónicas y la oratoria o práctica de los discursos. Critica las Retóricas por su carácter superficial y periférico frente a lo que debería ser su ocupación central: las formas de argumentar y los procedimientos que consiguen la persuasión, recursos

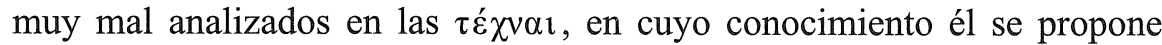
profundizar, intentando someterlos a principios lógicos que garanticen la veracidad ${ }^{10}$. Veamos la solución aristotélica a las críticas platónicas señaladas.

\section{El entimema como compromiso entre lo sỉ kós y la verdad}

Para Aristóteles la labor fundamental de la Retórica es la de proporcionar medios para argumentar, entendiendo estos medios en sus diferentes facetas: distintos procedimientos lógicos, material temático que pueda ser usado como premisas de entimemas, distintas funciones de cada recurso, etc. No debe extrañarnos, por tanto, que la mayor parte de su Retórica se dedique a ello, ni que al hablar de las partes del discurso, como veremos, las reduzca básicamente a introducción y argumentación. No cabe duda de que en este terreno su obra supuso un gran avance respecto a las retóricas anteriores en las que había una gran falta de sistematicidad y claridad en los procedimientos argumentativos ${ }^{11}$. Es evidente que nadie estaba mejor capacitado para esta tarea que el propio Aristóteles que sentó, parece que paralelamente al desarrollo de la Retórica, las bases de su Lógica ${ }^{12}$.

10 Arist., Rh. 1354a13-28: «Los que han compuesto Artes acerca de los discursos, ni siquiera ... han proporcionado una parte de tal arte, pues ... nada dicen de los entimemas, que son el cuerpo de la persuasión, y más bien se ocupan, las más de las veces, de cuestiones ajenas al asunto. Porque, en efecto: el mover a sospecha, a compasión, a ira y a otras pasiones semejantes del alma no son propias del asunto ... Nada compete al litigante fuera de mostrar que el hecho es o no es así y si aconteció o no aconteció», en la versión de Racionero.

11 El mejor ejemplo en este sentido es la obra de Anaxímenes, la llamada, Rhetorica ad Alexandrum. Goebel traza de forma magistral la evolución de los medios argumentativos.

12 Existe una relación profunda entre la lógica y la retórica, hasta el punto que se han separado distintas etapas compositivas de la Retórica en función de su relación con la lógica aristotélica, sobre este particular véase sobre todo Solmsen, sobre la base de Kantelhardt. Sobre toda esta cuestión es muy útil la discusión de Racionero, pp. 38-46. 
Los entimemas no son otra cosa que silogismos retóricos ${ }^{13}$. La diferencia fundamental con el lógico estriba en que el entimema no procede a partir de una verdad universal, sino de probabilidades o verosimilitudes ( $\varepsilon$ i kó $\tau \alpha)^{14}$. Es decir, parte de una premisa mayor - no suele expresarse por innecesaria, ya que se supone que el auditorio la asume ${ }^{15}$ - que es sólo probable ${ }^{16}$, por lo que la conclusión también lo es ${ }^{17}$; por ello es útil para convencer o persuadir al auditorio de un discurso pero no para establecer la verdad según los requisitos de la lógica. Suponen un intento de dar respuesta a la exigencia platónica en favor de la verdad, ya que son un tipo de argumentos verificables, en los que la parte contraria puede técnicamente descubrir y desmontar los paralogismos y así deben entenderse las Refutaciones sofisticas aristotélicas. Según eso, un discurso que consistiera básicamente en una argumentación construida sobre entimemas, podría someterse a una comprobación del valor lógico de sus argumentos, y por tanto de su veracidad.

Nuestra pregunta es, ¿Aristóteles en este terreno intenta modificar la realidad de los discursos o se está limitando en buena medida a interpretar y comprender los procedimientos argumentativos que usan? Creemos que sólo propone modificar la realidad (es decir, tiene una actitud normativa) en cuanto que pretende reducir el proceso de persuasión del discurso sólo a procedimientos basados en razonamiento lógico o entimemas, pero no intenta desde luego imponer un recurso persuasivo nuevo. Por un lado, en el terreno de la teoría retórica, reelabora material retórico preexistente so-

13 Desde un punto de vista formal, los dos recursos lógicos que propone Aristóteles en su Retórica son el entimema y el ejemplo. No cabe duda de la preeminencia del primero en cuanto que el ejemplo funciona muchas veces como elemento del entimema, $c f$. Hood, pp. 48-9. Solmsen sostiene que en una primera fase Aristóteles sólo consideraría el entimema como medio de prueba, posteriormente incluiría el ejemplo. Una discusión sobre el entimema y sus definiciones en Aristóteles puede verse en los dos trabajos de Burnyeat.

14 El mejor estudio sobre el entimema en Aristóteles es el de Ryan, con una rica discusión sobre las posturas anteriores. Rechaza en una discusión interesante que el carácter probable de las premisas sea lo que lo defina propiamente. Lo importante, si se entiende bien a Aristóteles, es que resulte convincente. Véase también Burnyeat 1996.

15 Es falsa la afirmación muy difundida que dice que el entimema es un silogismo al que le falta una de sus tres partes, aunque sea verdad que muchos entimemas carezcan de una de ellas. Cf. Hood que la denomina "assumed". Burnyeat 1994 explica cómo la idea de que el entimema es un silogismo al que le falta una de sus partes pudo originarse en la interpretación estoica de la lógica aristotélica.

16 Es clara la equiparación entre "probable" y ei kóc: Arist., Rh. 1357a34.

17 Sobre la validez lógica del entimema, véase el interesante trabajo de Burnyeat 1996. 
bre lo probable o verosímil ( $\varepsilon i$ kós), ya considerado, como es bien sabido, desde la Retórica siciliana ${ }^{18}$, y, por otro, se limita a teorizar sobre formas de argumentación existentes en gran profusión y extensión en los propios discursos ${ }^{19}$. Si eso es verdad, los discursos de los oradores anteriores a la Retórica aristotélica deben tener ya una argumentación construida a base de razonamientos lógicos basados en la probabilidad (para Aristóteles, entimemas).

Hemos estudiado las formas y funciones de los entimemas de los discursos, entendidos en clave aristotélica, en otros lugares ${ }^{20}$, pero creemos útil explicar brevemente cómo son los razonamientos oratorios que después Aristóteles designará como entimemas ${ }^{21}$. Para facilitar la tarea nos centramos en un discurso cualquiera de Lisias, concretamente el 3, Defensa contra Simón, como ejemplo de un discurso compuesto con anterioridad a la Retórica aristotélica. Se trata de una acusación de $\tau \rho \alpha \tilde{v} \mu \alpha \quad \dot{\varepsilon} \kappa$ $\pi \rho o v o$ í $\alpha \varsigma^{22}$ vista ante el Areópago. Simón acusa al cliente de Lisias de ser el responsable de una pelea callejera que acabó con lesiones de los implicados; el motivo del altercado era conseguir los favores sexuales del joven Teodoto para lo cual Simón afirma que le habría pagado 300 dracmas a modo de contrato; el cliente de Lisias, por su parte, sostiene que el joven estaba voluntariamente con él. Aunque en el proceso se manejan testimo-

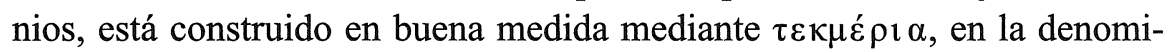

18 Un reflejo de la teoría habitual sobre el particular en Anax., Rh. Al. 7.4-6, 1428a. Es muy útil sobre este problema el cap. I de Goebel.

19 Trevett, pp. 374-5 demuestra que Aristóteles conocía todo tipo de discursos pero probablemente sólo disponía del texto de los discursos epidícticos porque eran los únicos que circulaban por escrito; así explica que cite muchas más veces discursos epidícticos. Con todo existen alusiones claras a textos de Lisias.

20 Para Lisias lo hemos demostrado en nuestros trabajos de 1996 para Lisias y 1994(b) con ejemplos de varios oradores.

21 Aunque hemos tratado en los lugares indicados las formas de los entimemas en extensión, conviene advertir que, a partir de los ejemplos del propio Aristóteles, una frase como «Está enfermo porque tiene fiebre» $(R h .1357 \mathrm{~b} 15)$ es un entimema, igual que «Ira mortal no alimentes, tú que eres mortal» ( $R h .1394 \mathrm{~b} 23)$, o «Si la guerra es la causa de los presentes males, es preciso que se enmienden con la paz» ( $R h$. 1397a11).

22 Vianello de Córdova ha hecho un exhaustivo y detallado estudio sobre todo lo que concierne al caso. Carey, pp. 86-92 nos ofrece también una presentación muy clara. Sobre el tipo de causa, cf. Todd, pp. 269 ss. Existe una polémica sobre si se trata de una $\gamma \rho \alpha \varphi \eta ́$ o una $\delta$ í $\uparrow$, cf. Vianello de Córdova, n. 10, pp. 13-14. Pueden ser útiles para contextualizar el caso los trabajos de Cohen. 
nación de Lisias ${ }^{23}$, es decir, razonamientos por lo عỉ kó $\varsigma^{24}$ (entimemas en la terminología aristotélica ${ }^{25}$ ). Es fundamental en la argumentación demostrar que no existió el contrato entre Simón y Teodoto; como indicios probables ${ }^{26}$ de que Simón miente a este respecto se aduce que no llamó a testigos y que se comportó de forma ultrajante, violenta, etc. Se trata de un entimema aristotélico basado en una premisa mayor no expresa ${ }^{27} \mathrm{del}$ tipo "los que hacen un contrato económico intentan ejecutarlo aportando testigos y no precisan de violencia", la premisa menor es "Simón no lo intentó ejecutar mediante testigos y ejerció violencia", siendo la conclusión que "el contrato no existió y Simón miente" ${ }^{28}$. De forma parecida se van formulando otros entimemas en la argumentación. No pudo hacer un contrato por 300 dracmas cuando declaraba una fortuna de 250 dracmas ${ }^{29}$. No existió el contrato y mucho menos le devolvimos las 300 dracmas después de la pelea, como afirma, porque no es $\varepsilon i \kappa^{\prime} \varsigma^{30}$ que uno devuelva el dinero que ha motivado una refriega cuando más enfrentadas están las partes. Es absolutamente inverosímil, dice, esa afirmación de Simón pero la formula para distraer del punto central en discusión y para justificar su

23 Así se usa la palabra en el propio discurso en los $\S \S 23$ y 45.

24 Bateman, citado por Vianello de Córdova, ha hecho un estudio pormenorizado de sus argumentos.

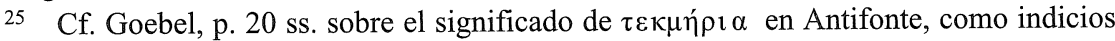
que se usan en argumentos de probabilidad, coincidente plenamente con el que hace aquí Lisias. Como demuestra Goebel este significado tiene bastante que ver con el de Anaxímenes y muy poco con el de Aristóteles.

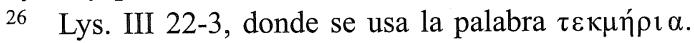

27 Ryan p. 61 ss. reacciona contra la posibilidad de reconstruir los entimemas tal y como se hace en este trabajo porque así se destruye su capacidad de convicción que es su aspecto determinante. Sin embargo de cara a su estudio nos parece un procedimiento casi imprescindible.

28 Como señala Ryan, p. 69 ss., lo importante del entimema no es que sea lógicamente válido sino que resulte convincente en el momento en que se usa.

29 Lys. III 24. La premisa mayor no expresa y de carácter probable sería: «no se hacen contratos por un monto económico superior a la fortuna de uno».

30 Lys. III 25 donde se usa el término eỉ kóc. La premisa mayor no expresa y de carácter probable sería: «Nadie devuelve el dinero de un contrato cuando se están produciendo peleas callejeras con la otra parte, si no existe una fuerza mayor»; premisa menor: «Se produjeron peleas callejeras y no existía fuerza mayor»; conclusión: «No le devolvimos el dinero». Pero otra conclusión es que Simón miente al afirmar algo contradictorio con la conclusión. Se trata de un entimema refutativo: La afirmación del contrario es contradictoria con la conclusión a la que se llega aplicando una premisa menor perfectamente establecida y una mayor en la que todo el mundo está de acuerdo. 
comportamiento violento ${ }^{31}$. No quedó malherido porque siguió corriendo y persiguiendo ${ }^{32}$, como lo corroboran los testigos. No fui a su casa a amenazarle y no actué con premeditación, pues no es creíble ( $\pi \imath \sigma \tau o ́ \varsigma$ ) que hubiera elegido ese momento para enfrentarme con él, en pleno día y cuando él estaba con muchos de sus amigos ${ }^{33}$. El que perseguía y buscaba hacer daño era él ${ }^{34}$. Tardó cuatro años en denunciarme cuando los despechados de amor buscan vengarse en el momento (yo no lo denuncié a él por vergüenza dada la naturaleza del asunto) ${ }^{35}$. Desde otro punto de vista no es

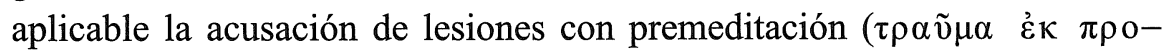
vo í $\alpha$ ) por la que me arriesgo a ser exilado porque ¿quién es tan ingenuo que tras planear atacar con premeditación reciba él mismo los golpes? ${ }^{36}$,

31 Lys. III 26. Aquí intenta razonar la motivación de un comportamiento tan fuera de la lógica. La premisa mayor no expresa y de carácter probable sería: «Los que se comportan con violencia disfrazan su comportamiento mediante falsas imputaciones, muchas veces inverosímiles». La menor es que a todas luces las imputaciones que hace Simón son absolutamente falsas. La conclusión es que Simón busca disfrazar su comportamiento violento. Ejemplo de silogismo de segunda figura de escaso valor lógico.

32 Lys. III 27. La premisa mayor no expresa y de carácter probable sería: «los malheridos se quedan quietos y no corren», la menor es «corrieron más de cuatro estadios, cosa de la que tengo testigos»; la conclusión implícita es que no estaba malherido. Esto es contradictorio a la afirmación de Simón de que estaba malherido, por tanto Simón miente. Es un nuevo ejemplo de entimema refutativo, como el del $\S 25$.

33 Lys. III 29: La premisa mayor no expresa y de carácter probable sería: «Cuando se actúa con premeditación para causar lesiones a alguien se evitan testigos molestos y amigos que protejan a la víctima». El propio Simón dice que acudí a su casa de día y cuando estaba reunido con todos sus amigos. La conclusión es que es falso que existiera premeditación y que Simón miente. Se refuta al adversario porque hace al tiempo dos afirmaciones que son contradictorias respecto a la premisa mayor, por otra parte, ninguna de las dos afirmaciones las comparte el cliente de Lisias.

34 Lys. III 36. En este caso tenemos todos los componentes, la premisa mayor aparece expresa («¿A quiénes hay que considerar responsables de los hechos, a los que persiguen o a los que huyen? Yo creo que está claro para todos que huyen los que están atemorizados por su propia persona y que persiguen los que quieren hacer algún daño»); la premisa menor es que los perseguidores eran Simón y sus amigos, afirmación que se hace en el $\S 35$. Por tanto, los que pretendían hacer daño eran Simón y sus amigos y Simón miente al declarar que el atacante era el cliente de Lisias.

35 Lys. III 39. En este caso, de nuevo, sí está expresa la premisa mayor: «los demás cuando están enamorados y se ven privados del objeto de su deseo y están desfallecidos, encolerizados buscan vengarse inmediatamente»; sí está expresa la menor, pero la conclusión debe suplirse por el contexto. A este entimema se asocia otro en sentido contrario que justifica que el orador no denunciara a Simón debido a su carácter vergonzoso.

36 Lys. III 41-2. La premisa mayor no expresa sería: «Los que actúan con premeditación planean su ataque con detalle y no resultan heridos en la refriega». La premisa menor 
sólo puede aplicarse cuando hay intención de matar. Desde luego que esa acusación no se usa para todas las peleas que se producen por la bebida, por rencillas amorosas por jovencillos o prostitutas, por insultos, etc.

Aristóteles justifica el carácter probable de los entimemas argumentando que no hay espacio o tiempo en un discurso para remontarse a la verdad universal, ni el propio auditorio lo admitiría ${ }^{37}$. Más allá de esta razón, se opera con probabilidades debido a la propia naturaleza de los temas de que se ocupan los discursos judiciales que gira fundamentalmente en torno al comportamiento humano sobre el cual muy difícilmente se pueden hacer afirmaciones universales o necesarias. Aristóteles es consciente de este hecho. Cuando analiza en el libro I, al referirse al discurso judicial, los motivos del comportamiento humano, distingue los comportamientos voluntarios de los que no lo son. Sólo los involuntarios cometidos mediante violencia $(\beta i \alpha)$ o por naturaleza ( $\varphi v ́ \sigma ı \varsigma)$ son necesarios, ni siquiera lo son los involuntarios que se deben al azar $(\tau v ́ \chi \eta)^{38}$. Es evidente que sobre los voluntarios, entre los que distingue los racionales de los irracionales, no pueden formularse afirmaciones necesarias.

En el mismo pasaje, tras estudiar los distintos motivos del comportamiento, concluye que los hombres actúan por cálculo racional $(\lambda \circ \gamma 1 \sigma \mu o ́ s)$,

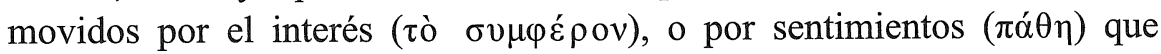
buscan el placer ${ }^{39}$. A continuación se pregunta sobre otros posibles componentes, como la edad, las actitudes ( $\check{\xi} \xi \varepsilon \varsigma$ ), etc. para explicar el comportamiento: no son causas de la conducta, pero con todo es verdad que a determinadas actitudes les suelen acompañar determinados comportamientos por lo que también «hay que considerar a qué tipo de personas acostumbra a acompañarles qué tipo de sentimientos» porque «si es joven o viejo, justo o injusto marca una diferencia» ${ }^{40}$, con lo que también los caracteres $(\eta \hat{\eta} \theta)$ son importantes en este terreno y también permiten formu-

contenida en la interrogación es «yo resulté herido». La conclusión es que no hubo premeditación.

37 Arist., $R h .1395$ b20-1396a4. Es muy útil la n. 280 de Racionero, p. 417. Cf. tb. $R h$. $1357 \mathrm{a}$.

38 Arist., Rh. 1368b35. Véase el comentario de Grimaldi 1980, p. 231 ss.

39 Arist., Rh. 1369a18 y $1369 \mathrm{~b} 7$ ss. Como el interés se ha estudiado a propósito del deliberativo, pasa a estudiar el placer, como lo más característico del judicial.

40 Arist., $R h .1369$ a24 y 27. Después en II 12-17 hablará del carácter de los jóvenes, viejos, maduros, afortunados, ricos y poderosos. 
lar premisas sólo de carácter probable. Promete que tratará más adelante con más detalle de los caracteres $(\eta \hat{\theta} \theta \eta)$ y de los sentimientos $(\pi \alpha ́ \theta \eta)^{41}$.

De acuerdo con esto, Aristóteles hace en el libro II un estudio del comportamiento humano que lo estructura en sentimientos ( $\pi \dot{\theta} \theta \eta$ II 2-11) y caracteres ( $\eta \hat{\theta} \theta \eta$, II 12-17), por ese orden, en cuanto que los primeros son determinantes y los segundos sólo intervienen como un factor añadido. Así interpretamos la finalidad y utilidad de estos capítulos que ha sido discutida reiteradamente ${ }^{42}$ : su función primordial, por tanto, es la de proporcionar premisas mayores verosímiles de especial utilidad para la oratoria judicial ${ }^{43}$.

Que en la práctica oratoria se razona a partir de probabilidades que tienen que ver con sentimientos o caracteres, lo podemos comprobar si nos fijamos ahora en las premisas mayores que hemos establecido en la reconstrucción de los entimemas de la argumentación del discurso de Lisias que estamos manejando. Así por un $\pi \alpha ́ \theta$ os, concretamente el miedo ( $\varphi o^{-}$ $\beta \circ \varsigma)^{44}$, huyen los que están atemorizados o por otro, el sentimiento de despecho, se genera cólera y deseo de venganza inmediato ${ }^{45}$. Por el $\tilde{\eta} \theta 0 \varsigma$ que caracteriza al acusado, propio de una persona un tanto timorata, no es verosímil que hubiera acudido con premeditación a casa de Simón para

41 Arist., $R h .1369 \mathrm{a} 30$ y $1369 \mathrm{~b} 15$. Al final, llegamos a un sistema para explicar el comportamiento en el que intervienen el cálculo $(\lambda \circ \gamma \iota \sigma \mu o ́ s)$ presidido por lo conveniente (para el que remite al cap. I 6), los sentimientos ( $\pi \alpha \dot{\theta} \theta \eta)$ presididos por el placer (remite a II 2-11) y los caracteres ( $\left.\eta^{\prime} \theta \eta\right)$, para los que remite a II 12-17, que no es muy diferente del de

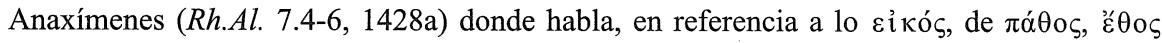
('costumbre') у ке́ $\rho \delta$ os ('interés').

42 Estamos de acuerdo en esto con Hellwig, p. 234 ss. Es muy claro en el mismo sentido el trabajo de Conley, aunque sólo lo aplica a los $\pi \alpha ́ \theta \eta$. J. Wisse, p. 38 ss. considera que ésta es una de sus funciones posibles, pero la primaria sería la de conocer al auditorio. Discute el problema con amplitud y cita las distintas posiciones.

43 Ha sorprendido a los comentaristas el punto de vista que adopta aquí Aristóteles, por ejemplo Racionero, n. 9, p. 310, por presentar las pasiones como un fenómeno físico-psicológico sin valor moral alguno, pero se entiende perfectamente si sólo se trata de proporcionar material al orador.

44 Nos referimos a la premisa mayor del entimema del $\S 36$. Cf. Arist., $R h$. 1382b-1383a, donde se contraponen las situaciones que producen temor (situación de peligro inminente) frente a las que producen confianza.

45 Premisa mayor del entimema del $\S 39$. Cf. Arist., Rh. 1379a donde se nos dice que se encolerizan los que desean algo y no lo consiguen y poco después en $1380 \mathrm{~b}$ que la cólera se aminora con el paso del tiempo. 
hacerle daño ${ }^{46}$. Sin embargo Simón tiene un carácter de canalla que explica su comportamiento violento una y otra vez ${ }^{47}$. Otras de las premisas mayores que hemos mencionado más que a un $\tilde{\eta} \theta$ o $\varsigma$ hacen referencia a costumbres, a hechos de comportamiento que son de observación habitual, como que los que hacen un contrato buscan ejecutarlo mediante testigos o que nadie devuelve el dinero después de una pelea, o que los violentos buscan disfrazar su comportamiento mediante falsas imputaciones contra sus víctimas, evitan actuar en presencia de testigos y son perseguidores más que perseguidos. Comprobamos que muchas de las premisas mayores se estructuran en torno a sentimientos $-\pi \alpha \dot{\theta} \theta \eta-y$ caracteres $-\ddot{\eta} \theta \eta-$. Son de hecho muy pocas las premisas de entimema del discurso citado que se salen de este marco ${ }^{48}$.

En definitiva, Aristóteles está sólo generalizando a partir de lo que ocurría realmente en los propios discursos. Su novedad y aportación radica en la conexión con la lógica del procedimiento argumentativo por lo عỉós, conexión que se hace a través del entimema, y en la importancia relativa en el conjunto de su obra retórica, puesto que, al entender como hemos señalado los caps. II 2-17, los libros I y II se dedican básicamente a proporcionarnos material para construir entimemas ${ }^{49}$. Intenta cambiar el centro de atención de la retórica pero ello no quiere decir que pretenda modificar fundamentalmente el proceso argumentativo de los discursos, aunque sí busca reducirlos exclusivamente a argumentación entimemática. Es nor-

$46 \S \S 41-2$, con la palabra $\varepsilon v ̉ \eta ́ \theta \eta \varsigma, ~ ' i n g e n u o '$, en el texto. La ingenuidad es el rasgo de carácter que tipifica al hablante. En la Retórica aristotélica los jóvenes son $\varepsilon$ v̉ $\theta \varepsilon 1 \varsigma$ (1389a16). Muchos de sus rasgos de carácter coinciden con los del orador de este discurso, aunque no sea joven: se dejan llevar por los deseos amorosos (1389a5), son confiados (1389a17), se avergüenzan con facilidad (1389a28). Recordemos que por vergüenza no denunció en su día a Simón, como hemos visto en el $\S 39$.

47 A Simón le son aplicables algunas de las premisas sobre el carácter de los viejos de

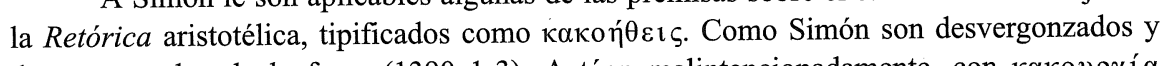

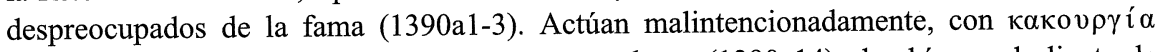
(1390a18). Se mueven por interés más que por deseo (1390a14), de ahí que el cliente de Lisias se pregunte cómo es posible que Simón pudiera enamorarse del muchacho, cuando lo de enamorarse corresponde a personas de carácter ingenuo. Cf. Arist., Rh. 1389a28.

48 Algunas son de naturaleza estrictamente lógica, como, por ejemplo, que el que sólo tiene 250 dracmas de fortuna no puede hacer un contrato por valor de 300 dracmas.

49 El libro I dedica sus tres primeros caps. a una teoría general de la argumentación y los medios de prueba y el resto a proporcionar material en función de los fines por los que se argumenta en cada uno de los géneros, mientras que el libro II lo proporciona en función de la conducta humana. 
mativo en cuanto que quiere que la teoría retórica cambie el centro de sus preocupaciones y añada la perspectiva lógica, pero es descriptivo en cuanto que sencillamente está dotando de un contenido lógico la realidad de los discursos, cuyas argumentaciones, ya en época anterior a Aristóteles, eran básicamente una serie de entimemas, como hemos demostrado. Además su teoría del entimema no es más que una reelaboración de lo verosímil ( $\varepsilon i$ kós), elemento que caracterizaba la Retórica desde la época de Córax y Tisias.

Debe quedar claro, por tanto, que cuando Aristóteles insiste en que la argumentación se basa en el entimema no está proponiendo que deba ser así, sino describiendo y descubriendo los secretos del proceso argumentativo generalizado en los discursos.

\section{La $\psi v \chi \alpha \gamma \omega \gamma i ́ \alpha$ en la Retórica aristotélica y en la práctica oratoria}

Existe un acuerdo general en el sentido de que Aristóteles intentó en su Retórica dar cumplimiento al deseo platónico, mencionado al principio de este trabajo, de que el rétor debía, como una de sus ocupaciones fundamentales y previas, conocer el alma ( $\left.\psi v \chi \eta)^{\prime}\right)$ de su interlocutor; la discusión estriba en determinar cuándo y cómo lo lleva a cabo.

A fin de no alargar, nos vamos a ceñir a discutir dos posturas de la crítica moderna. Hellwig ${ }^{50}$ considera que Aristóteles, buscando, una vez más, un compromiso entre Platón y la práctica retórica y oratoria del momento, sin desarrollar desde luego una Psicología como tal, sí hace hincapié en la importancia de la personalidad del hablante ( $\tilde{\eta} \theta 0 \varsigma)$ y de las reacciones

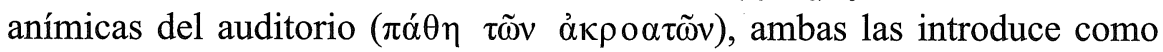
dos de los tres medios de persuasión $(\pi i \sigma \tau \varepsilon i \varsigma)^{51}$. Además, Aristóteles dentro de esta preocupación por la base psicológica explica el comportamiento por los $\eta^{\theta} \theta \eta$ y por los $\pi \alpha ́ \theta \eta$, lo que al tiempo sirve para proporcionar premisas de entimemas.

Wisse ${ }^{52}$ sin embargo, aunque está de acuerdo en que no tiene que ver el carácter del hablante como una de las tres $\pi$ í $\tau \varepsilon \imath \varsigma$ con los caracteres del

\footnotetext{
$50 \quad$ Pp. 233-248.

51 Arist., Rh. 1356a1-13.

52 Él mismo es consciente de la dificultad que entraña el hecho de que los auditorios no sean homogéneos, cosa que sería una condición necesaria para que fueran aplicables los $\eta \eta \theta \eta$ aristotélicos de II 12-17. No existe un auditorio público de sólo viejos o sólo jóvenes.
} 
libro II, considera que estos capítulos (II 12-17) cumplen con el deseo platónico relativo a la necesidad de una Psicología, en cuanto que su función primordial es la de permitir al orador acercarse a la psicología del auditorio, a su carácter, para poder influir más fácilmente en sus emociones o sentimientos. Posteriormente, en esta misma línea, Aristóteles habría añadido II 2-11 sobre los sentimientos, pensando sobre todo en el auditorio. Esta sería la forma en la que dio cumplimiento a los requerimientos de su maestro. Está claro que nuestra posición se acerca mucho más a la de Hellwig. Los capítulos dedicados a los caracteres (II 12-17) son útiles para proporcionar premisas de entimemas, por una parte para explicar el comportamiento y secundariamente para conseguir que el orador sea digno de confianza y el contrario no. No tienen desde luego mucho que ver con el conocimiento del auditorio, que por definición es variado, a menos que nos refiramos a situaciones de oratoria privada y no pública. De la misma forma los sentimientos (II 2-11) sirven para proporcionar premisas de entimemas que explican el comportamiento y, en otra dirección, para provocar sentimientos en el auditorio por vía entimemática.

Existe, por otra parte, una contradicción aparente entre el hecho de que Aristóteles condene el uso de los sentimientos al principio de su obra ${ }^{53}$, en un texto claramente normativo en relación a las otras Retóricas, y, luego, como estamos viendo, los considere entre los medios de prueba ( $\pi$ í $\sigma-$ $\tau \varepsilon \imath \varsigma)$ un poco más adelante. En general la solución que se ha dado a este conflicto residiría en una evolución del pensamiento aristotélico. La condena a los sentimientos de la introducción pertenecería a una etapa más platónica, por tanto más normativa, mientras que el segundo pasaje sería de una etapa posterior, más descriptiva, en la que se ha acercado más a la realidad de los discursos ${ }^{54}$. Existe otra solución más integradora que postula Grimaldi entre otros ${ }^{55}$, según la cual Aristóteles admitiría provocar sentimientos en el auditorio siempre y cuando se hiciera por procedimientos entimemáticos ${ }^{56}$. Ya hemos demostrado en otros lugares ${ }^{57}$, con ejemplos concretos de discursos, que esto es perfectamente posible y que no se

\footnotetext{
53 Arist., Rh. 1354a12-15.

54 Por ejemplo, Solmsen, o en fecha mucho más reciente Fortenbaugh, 1992, pp. 234-5, en un trabajo de sumo interés para la cuestión que tratamos.

55 Grimaldi, 1972, p. 68. Véase también su trabajo de 1980. En Wisse, p. 36 ss., puede encontrarse la discusión en detalle.

56 Sobre la relación entre sentimiento y racionalidad, véase el trabajo de Nussbaum.

57 Nuestros trabajos de 1994 y 1996.
} 
trata de un mero desiderátum aristotélico o de entimemas ideales en expresión de Wisse ${ }^{58}$. El discurso que nos sirve de ilustración, a pesar de ser pronunciado ante el Areópago, por lo que se aplica en él la restricción contra el uso de los sentimientos que comenta el propio Aristóteles ${ }^{59}$, contiene ejemplos de este tipo, uno en el proemio y otro en el epílogo: no deben despreciar al cliente de Lisias por haber sucumbido a los sentimientos amorosos que le han puesto en las dificultades en las que se encuentra ${ }^{60}$; es digno de compasión, pero por vía razonada o entimemática, en cuanto que ha cumplido con todas sus obligaciones respecto a la ciudad (militares e impositivas) y porque ha sido forzado a tratar de temas escabrosos para su propia reputación ${ }^{61}$.

Ahora bien, subsiste la dificultad de justificar la condena que se hace al uso de los sentimientos al principio de la Retórica, ¿qué sentimientos son los que pretende desterrar Aristóteles? Han de ser otros que los que se provocan mediante entimemas, tal y como hemos argumentado. Una vez más, si dirigimos nuestra mirada a los discursos lo podemos entender mucho mejor. Se trata de las apelaciones directas a los sentimientos de los jueces realizadas por medios no entimemáticos: súplicas en el proemio que invocan la benevolencia buscando crear un estado de ánimo en el auditorio favorable y súplicas en las que se apela a la compasión ('̌́ $\lambda \varepsilon \circ \varsigma$ ) o la cólera (ỏ $\rho \eta ́$ ), dependiendo que se trate de un discurso de acusación o

58 Wisse, p. 26.

59 Arist., $R h$. 1354a22. El propio orador se refiere a esta limitación en su epílogo, Lys. III 46. Sobre el particular, cf. Bearzot.

60 Lys. III 4, a partir de un entimema sobre una premisa mayor implícita: «Hay que indignarse sólo ante comportamientos indignos»; premisa menor: «Mi comportamiento no es indigno, sino que se debe al amor en el que todos caemos»; conclusión: «No debéis indignaros por mi comportamiento». Cf. Arist., Rh. 1378a10 ss. sobre el desprecio (ỏ $\lambda \_\gamma \omega-$ $\rho i \alpha)$ y sus formas, una de ellas el ultraje ( $\ddot{\beta} \rho \imath \varsigma)$, se define como «decir aquello por lo que siente vergüenza el afectado».

61 Lys. III 47-8. El razonamiento reposa en un entimema con una premisa mayor implícita del tipo: «Son dignos de compasión los que se ven envueltos en el peligro de ser expulsados de su patria a pesar de haber arriesgado la vida por ella y de haberse enfrentado a todas las $\lambda \varepsilon \imath \tau o v \rho \gamma i \alpha_{1}$, y los que se ven forzados a exhibir sus sentimientos amorosos». Premisa menor expresa: «Yo he hecho frente a mis obligaciones militares y a los impuestos de la ciudad y he tenido que exponeros por la denuncia de ése todos mis sentimientos». Conclusión: «Soy digno de compasión». Cf. Arist., Rh. 1386a17 ss.: se compadece uno de los conocidos, iguales en edad, costumbres, formas de ser, etc., todo aquello que uno teme referido a sí mismo. 
defensa, características del epílogo de los discursos judiciales ${ }^{62}$. Tenemos, además, referencias del empleo de otros procedimientos de naturaleza no verbal como hacer subir al estrado a familiares llorosos que con su actitud provocan la compasión ${ }^{63}$, en un recurso típico también de discurso judicial. En este caso no cabe duda del carácter normativo de Aristóteles al pretender desterrar un uso oratorio habitual contemplado también por las retóricas ${ }^{64}$. El motivo es bien claro, los discursos sólo deben contener elementos racionales o entimemáticos para poderlos someter a un proceso de verificación lógica. No es peligroso el uso de sentimientos siempre que estos dependan de un proceso deductivo, ésta parece ser la respuesta a la objeción de la introducción de su obra. Pero Aristóteles no pretende incorporar algo nuevo a los discursos, sino desarrollar más algo existente y suprimir las apelaciones directas a los sentimientos por ser irracionales. El discurso que manejamos como ejemplo no contiene estos elementos por haberse pronunciado en el Areópago.

Es más difícil entender qué es lo que pretende decir Aristóteles cuando habla del carácter del hablante como uno de los tres medios de prueba ( $\pi$ í $\tau \varepsilon \iota \varsigma$ ), aunque investigaciones recientes han hecho avanzar mucho nuestra comprensión ${ }^{65}$. Se ha demostrado que al hablar del carácter del

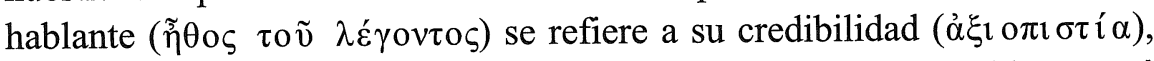
es decir consiste en que el auditorio se convenza de que el hablante está diciendo la verdad, lo cual se consigue haciendo que aparezca como dotado de sensatez ( $\varphi \rho o ́ v \varepsilon \sigma \imath \varsigma$ ), virtud ( $\alpha \rho \varepsilon \tau \eta ́)$ y benevolencia $(\varepsilon v ̛ v o \iota \alpha)^{66}$. Debe lograrlo argumentativamente y no porque el auditorio tenga previamente una opinión de él, buena o mala ${ }^{67}$. Es un procedimiento persuasivo más útil en deliberativos que en judiciales, según el propio Aristóteles ${ }^{68}$. Es algo distinto a que el orador resulte simpático al auditorio, puesto que esto equivaldría a provocar un sentimiento. Pretende separar lo relativo al hablante de lo que afecta a los oyentes. Sin embargo, casi nadie lo ha en-

62 Cortés Gabaudan 1986, p. 107 ss.

63 Hemos estudiado el fenómeno en el trabajo mencionado en la nota anterior, pp.

131-2, cf. P1., Ap. 34c, Isoc. XV 321, Lys. XVII 24, D. XXI 186, XXXVIII 27.

64 Concretamente existe un apartado dedicado a las peticiones en Anax., Rh. Al. 19.1

1.433 b17-28, comentado en p. 117 ss. de la ob. cit. en nota anterior.

65 Fortenbaugh, 1988 y 1975. Wisse, pp. 32-4. También Schütrumpf.

66 Arist., $R$. 1378a 6-8.

67 Arist., Rh. 1356a 1-13.

68 Arist., Rh. 1377b 28-31, cf. Fortenbaugh, 1992. 
tendido así y se mezclaron los dos campos, como ocurre en Cicerón que habla de beneuolentiam conciliare ${ }^{69}$, en Dionisio de Halicarnaso para el cual se confunde con la etopeya ${ }^{70}$, etc. y también en la mayor parte de los estudiosos modernos ${ }^{71}$. Todos ellos consideran que la credibilidad del hablante consiste en hacer que éste resulte simpático. Con ello en realidad vuelven a la situación prearistotélica tal y como nos la refleja Anaxímenes ${ }^{72}$. Para cualquier lector de oratoria ática es claro que los oradores se esfuerzan por convencer a su auditorio de que su comportamiento es propio de un carácter sensato, bien intencionado y virtuoso, a diferencia de lo que ocurre con los contrarios. Aristóteles y los oradores se han dado perfectamente cuenta de la importancia que tiene este factor en el proceso persuasivo. Si no nos produce credibilidad el hablante, no confiaremos en nada de lo que nos diga. Este factor todavía es más fuerte en el género deliberativo, como señala Aristóteles ${ }^{73}$, pues en muchas ocasiones la parte contraria se limita mediante la $\delta 1 \alpha \beta o \lambda \eta ́$ a descalificar al hablante sin entrar en sus argumentos. En lo que insiste nuestro rétor es que debe conseguirse la credibilidad propia y disminuirse la del contrario por vía razonada, no de forma irracional con alabanzas a uno mismo o insultos al contrario.

El discurso que estamos usando como ejemplo es muy claro a este respecto. El orador tiene verdadera necesidad de justificar la rectitud de su carácter y comportamiento, dada la naturaleza del caso. Porque está convencido de la justicia de su comportamiento, nos dice, le basta con que emitan una sentencia justa ${ }^{74}$. También por su carácter sensato y virtuoso siente vergüenza de tener que narrar el asunto del caso, pero se ve forzado a ello y no va a esconder nada ${ }^{75}$. Es mejor y más sensato quien mejor en-

\footnotetext{
69 Cic., De orat. II 182.

70 D.H., Lys. VIII 19.

71 Wisse, pp. 43-64, dedica un apartado al particular.

72 Fortenbaugh, 1992, pp. 226-7.

73 Arist., $R h$. 1416a 4-ss., a continuación de lo relativo al proemio de deliberativos.

74 Lys. III 1 que esconde un entimema con una premisa mayor implícita «Los que se han comportado con justicia confían en jueces justos»; premisa menor: "Yo me he comportado con justicia y vosotros sois justos»; conclusión: «Confío en vosotros y la justicia de vuestra sentencia». Por otra parte, está claro que comportarse con justicia es indicio de un carácter sensato, virtuoso, bien intencionado.

75 Lys. III 2, a partir de un entimema con una premisa mayor implícita del tipo: «Son sensatos los que sienten vergüenza de contar sus líos amorosos y los que cuentan toda la verdad»; premisa menor explícita: «Yo me avergüenzo pero me veo forzado a contaros mis
} 
frenta las desgracias ${ }^{76}$, algo aplicable al orador. Simón, por el contrario, tiene un comportamiento lleno de úßpı $\varsigma$ y violento, por lo que «no respetó la inviolabilidad del gineceo y atentó contra las mujeres de mi casa que son gente recta» ${ }^{77}$, nos dice a continuación. «Por vergüenza y para no ser motivo de burla soporté sin denunciarlas las afrentas de Simón ${ }^{78}$ e incluso me marché de la ciudad». Así es como se refuerza la credibilidad del hablante en esta primera parte del discurso que engloba el proemio y la narración ${ }^{79}$. Por su parte, la argumentación, básicamente refutativa, insiste nada más abrirse en la falta de credibilidad de Simón: es perjuro y mentiroso ${ }^{80}$, insultos que se repiten cada poco, y que sólo en contadas ocasiones se comprueban de forma racional (porque no ha llamado a testigos ${ }^{81} \mathrm{O}$ porque afirma que ha hecho un contrato por una cantidad superior a la fortuna que ha declarado ${ }^{82}$ ), procedimiento éste que no resultaría del agrado de Aristóteles. Y para cerrar la argumentación se busca un golpe de efecto sobre la falta de credibilidad de Simón a partir de un comportamiento aje-

líos amorosos, lo haré con toda la verdad»; conclusión implícita: «Soy sensato». Cf. Arist., $R h$. 1383b21: es motivo de vergüenza la lujuria: tener trato carnal con quien no se debe, cuando no se debe.

76 Lys. III 4, con la premisa mayor expresa, la menor y la conclusión se reconstruyen por el contexto.

77 Lys. III 6. La ű $\beta \rho ı \varsigma$ de Simón se demuestra a partir de una premisa mayor implícita del tipo: «Quienes se comportan con ü $\beta \rho \imath \varsigma$ son violentos, no respetan los gineceos, se emborrachan, etc.». Estas circunstancias estaban en el comportamiento de Simón, pues estaba borracho, entró violentamente, invadió el gineceo, no quiso marcharse, etc., que desde el punto de vista silogístico serían las premisas menores. La conclusión no hace falta explicitarla. Sobre la ű $\beta \rho 1 \varsigma$ y quienes la sufren, Arist. $R h .1378 b 23$ ss. Este comportamiento se contrapone con la rectitud de sus familiares femeninos que se comprueba por la vergüenza de ser vistas. De forma implícita es aplicable esta rectitud al orador.

78 Lys. III 9-10. Este sentimiento de vergüenza corresponde a una persona recta que teme por su reputación, frente a la desvergüenza pública de Simón. De nuevo aquí hay un entimema escondido, sobre una premisa mayor de quiénes son los que resienten vergüenza, ya comentado en una nota anterior.

79 De la división en partes de este discurso hablaremos al final de este trabajo.

80 Lys. III 21. Sencillamente se afirma. La convicción de que esto es verdad la lograrán por vía racional los jueces, si se convencen que sus declaraciones son mentirosas, es decir por el conjunto de la refutación.

81 Lys. III 23. Aquí si está implícito un entimema en esta acusación, premisa mayor implícita: «Quienes dicen verdad se apoyan en testimonios»; la menor: «No se apoya en testimonios»; conclusión: «es un mentiroso». Este razonamiento se hilvana con una serie de insultos descalificantes para el adversario.

82 Lys. III 24, en un entimema ya comentado. 
no al caso: es un canalla capaz de todo como se demuestra por su enfrentamiento con el taxiarca Laquetas que resultó en su expulsión de la expedición a Corinto ${ }^{83}$, es decir, no fue capaz de cumplir con sus obligaciones militares y ciudadanas. Simón es, pues, una malísima persona con un comportamiento execrable, capaz de entrar violentamente en las casas ajenas, de perseguir a alguien para violentarlo y llevárselo a la fuerza ${ }^{84}$. A diferencia del orador que cumplió con todas sus obligaciones ciudadanas, incluido el pago de impuestos ${ }^{85}$.

\section{Las partes del discurso}

Volviendo de nuevo a las críticas platónicas del inicio, nos falta por ver cómo realiza Aristóteles la exigencia de su maestro en el sentido de que el discurso debe ser coherente ${ }^{86}$. Con ello pasamos ahora al libro III, concre-

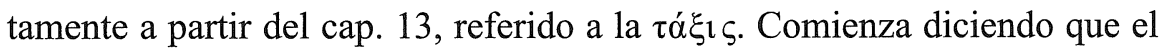
discurso sólo tiene dos partes necesarias, $\pi \rho$ ó $\theta \varepsilon \sigma \iota \varsigma$ (propositio de los latinos o decir muy brevemente cuál es el tema y nuestra postura sobre el mismo) ${ }^{87}$ y $\pi$ í $\sigma \tau \iota \varsigma$ o argumentación. A continuación ridiculiza las clasificaciones retóricas que enumeran muchas partes, en una crítica que se remonta a Platón ${ }^{88}$. Luego hace un tratamiento específico de cuatro partes:

83 Lys. III 45. Aquí se corrobora el carácter de Simón por vía de ejemplo más que por vía deductiva.

84 Lys. III 46. A modo de resumen recoge las acusaciones que considera ya probadas para convencernos del carácter de Simón. El razonamiento descansa en un entimema de este tipo. Premisa mayor expresa de forma indirecta: «Los canallas son capaces de comportamientos violentos una y otra vez». Premisa menor expresa de forma indirecta: «Simón tuvo varios comportamientos violentos: se enfrentó al taxiarca, penetró con violencia en mi casa, nos persiguió y se llevó a la fuerza al muchacho». La conclusión implícita es que «Simón es un canalla».

85 Lys. III 47-8. En un último entimema ya en el epílogo, tópico en los discursos de la época. Premisa mayor implícita: «Sólo los buenos ciudadanos se arriesgan por su ciudad y pagan todas las $\lambda \varepsilon \imath \tau o v \rho \gamma i ́ \alpha \imath$ ». Premisa menor: «Yo lo he hecho». Conclusión: «Soy un buen ciudadano [digno de confianza]». El mismo pasaje lo hemos citado como entimema que justifica la compasión de los jueces.

${ }^{86}$ Iglesias Zoido en el cap. 3 de su I parte, «El discurso y sus partes: el papel de la $\pi$ í $\tau \imath \varsigma »$, pp. 29 ss., desarrolla un planteamiento muy parecido al que hacemos aquí, aunque desde otro punto de vista.

87 Cf. Cortés Gabaudan 1984.

88 Pl., Phaedr. 266e ss. 


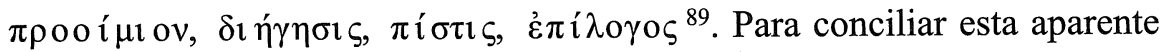
contradicción se ha hablado de que se conjuga una visión estricta sobre la retórica, propia de la Academia, que pretende limitar todo a la argumentación ${ }^{90}$, con una más cercana a la realidad. Según eso Aristóteles adoptaría aquí una postura claramente normativa.

Pues bien todo queda explicado si uno considera que Aristóteles está pretendiendo exclusivamente describir la realidad de los discursos, que es variable según los géneros. Dado que la narración es una parte claramente innecesaria en los deliberativos y que, como tal, no se encuentra en estos discursos, y que la narración se fragmenta en los epidícticos y a veces en los judiciales, se hace evidente que no se puede considerar que sea una de las partes necesarias, aunque sí es desde luego una parte habitual de los judiciales. Por otro lado, dado que el proemio no aparece de hecho en muchos deliberativos o sólo en una forma muy breve ${ }^{91}$ o como $\delta 1 \alpha \beta o \lambda \eta \dot{n}$, y que, como argumenta el propio Aristóteles, es innecesario en determinados judiciales, tampoco se puede considerar parte necesaria. En cuanto al epílogo ocurre algo semejante, en los deliberativos más que el epílogo de contenido emocional propio de judiciales, lo que tenemos, en el mejor de los casos, es un sencillo y breve resumen de los argumentos. Ahora bien, no deja de ser verdad que sea necesario desde un punto de vista retórico hablar de las cuatro partes que considera Aristóteles (proemio, narración, argumentación y epílogo), en cuanto que aparecen como tales normalmente en judiciales. Así ocurre en el discurso de Lisias que hemos seguido como ejemplo de nuestra exposición ${ }^{92}$. Parece claro que Aristóteles no está, una vez más, intentando cambiar nada de la práctica oratoria, sencillamente pretende describirla. Otra cosa es que critique las otras retóricas que aplican una teoría de las partes propia del género judicial a otros gé-

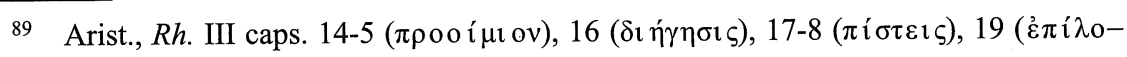
$\gamma \circ \varsigma)$.

90 Por citar dos ejemplos de este análisis, baste citar a Racionero, nota 277 de p. 555 o a Fortenbaugh 1992, pp. 234-6.

91 Para el caso de los discursos deliberativos de Tucídides, nos remitimos a F. Romero Cruz, Para el caso de Demóstenes bastaría citar el análisis de partes de los discursos que hace F. Blass.

92 Proemio en $\S \S 1-4$; narración $\S \S 5-20$, aunque se citen testigos para corroborar los hechos expuestos en $\S 14$; argumentación $\S \S 21-45$, planteada sobre todo como refutación de las acusaciones del contrario, como es propio en un discurso de defensa; epílogo $\S \S$ 46-48. En Vianello de Córdova se discuten en detalle las partes de este discurso. 
neros en los que es inaplicable, debido a un trasvase de normativa retórica, como hemos demostrado en otro lugar ${ }^{93}$.

\section{Conclusión.}

Gracias a este recorrido por la Retórica de Aristóteles creemos haber demostrado que debemos hacer una lectura de esta obra que tenga en cuenta la finalidad para la que está compuesta: descubrir los mecanismos persuasivos de los discursos reales para poderlos aplicar conociendo sus secretos. A partir de este hecho, que suele olvidarse, ya que se considera la Retórica como un edificio teórico de carácter normativo, se aclaran, como hemos pretendido demostrar, muchas cuestiones que afectan a temas muy debatidos en la comprensión de esta obra compleja. Concretamente:

1. La argumentación de los discursos con anterioridad a Aristóteles se basaba en entimemas, en el sentido que da al término en su Retórica, cuyas premisas aludían en muchas ocasiones a sentimientos y caracteres, a partir de generalizaciones probables ( $\varepsilon \hat{i} \kappa o ́ \tau \alpha)$ del tipo «las personas embargadas de tal sentimiento se comportan así», o «las personas de tal carácter se comportan de tal manera» con las que se pretendía explicar comportamientos. A partir de aquí defendemos que la función básica de los capítulos del libro II de la Retórica 2-11 (sobre los $\pi \alpha ́ \theta \eta$, sentimientos) y $12-17$ (sobre los $\eta \hat{\theta} \theta \eta$, caracteres) es proporcionar material para construir razonamientos de ese tenor.

2. Mediante entimemas en sentido aristotélico se provocaba ya en discursos anteriores a la Retórica sentimientos en el auditorio y se aumentaba la credibilidad del orador. Es verdad que para este cometido puede usarse parte (sólo una pequeña parte) del material del libro II al que hemos aludido en el punto anterior.

3. La condena que hace Aristóteles al uso de los sentimientos se refiere a los que son provocados por apelaciones directas a los jueces, no a los sentimientos excitados por vía entimemática o racional.

4. En cuanto al número de las partes del discurso el conflicto aparente sobre el número de partes que consideraba Aristóteles se soluciona fácilmente si nos damos cuenta que los deliberativos propiamente sólo tenían

93 Nuestro trabajo de 1994a. 
dos partes ( $\pi \rho o ́ \theta \varepsilon \sigma 1 \varsigma$ o propositio y argumentación), mientras que la mayor parte de los judiciales, aunque no necesariamente, contaban con cuatro. Por ello la Retórica hace el estudio de esas cuatro partes, aunque sólo dos basten para constituir un discurso.

\section{BIBLIOGRAFÍA CITADA}

Bateman, J.J. 1958: A Study of the Arguments in the Speeches of Lysias, diss. Cornell University.

Blass, F. 1893: Die attische Beredsamkeit, III.1 Demosthenes, Leipzig (=1962).

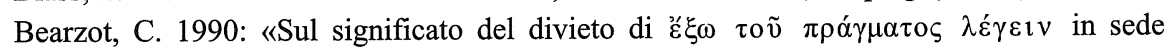
areopagitica», Aevum 64, pp. 47-55.

Burnyeat, M.F. 1994: «Enthymeme: Aristotle on the Logic of Persuasion» en D.J. Furley \& A. Nehamas, Aristotle's Rhetoric. Philosophical Essays, Princeton, N. Jersey (Princeton U.P.) 1994, pp. 3-55.

- 1996: «Enthymeme: Aristotle on the Rationality of Rhetoric», en A. Oksenberg Rorty, Essays on Aristotle's Rhetoric, Berkeley, pp. 88-115.

Carey, Ch. 1989: Lysias. Selected Speeches, Cambridge.

Cohen, D. 1991 a: «Sexuality, Violence, and the Athenian Law of Hybris», G\&R, 38, pp. 172-188.

- 1991 b: Law, Sexuality and Society, the Enforcement of Morals in Classical Athens, Cambridge.

- 1995: Law, Violence and Community in Classical Athens, Cambridge.

Cole, Th. 1991: The Origins of Rhetoric in Ancient Greece, Baltimore-Londres.

Conley, Th. 1982: «Páthe and pisteis: Aristotle 'Rhet'. II 2-11», Hermes, 110, 300-315.

Cortés Gabaudan, F. 1986: Fórmulas retóricas de la oratoria judicial ática, Salamanca.

- 1989: «Origen de las funciones del proemio y de las cualidades de la 'narración' « en Actas del VII Congreso Español de Estudios Clásicos, II, 135-140, Madrid.

- 1994 a: «El trasvase entre géneros oratorios en las primeras retóricas» en Actas del VIII Congreso Español de Estudios Clásicos, II, 131-138, Madrid.

- 1994 b: «Formas y funciones del entimema en la oratoria ática», CFC (Est. grie. e ind.) 4, 205-225.

- 1996: «La interpretación del texto: Lisias 24. (Lectura aristotélica de la argumentación)», Didáctica del Griego y de la Cultura Clásica, eds. F.L. Lisi, J. Ureña y J.C. Iglesias, Cáceres, pp. 96-112.

Fortenbaugh, W.W. 1975: Aristotle on Emotion, Londres.

- 1988: «Benevolentiam conciliare and animos permovere: Some Remarks on Cicero's De Oratore II 178-216», Rhetorica 6, pp. 259-273.

- 1992: «Aristotle on Persuasion through Character», Rhetorica 10, 207-244.

Goebel, G.H. 1983: Early Greek Rhetorical Theory and Practice. Proof and Arrangement in the Speeches of Antiphon and Euripides, Wisconsin.

Grimaldi, W.M.A. 1972: Studies in the Philosophy of Aristotles's Rhetoric, Wiesbaden.

- 1980: Aristotle, Rhetoric I. A Commentary, N. York. 
Hellwig, A. 1973: Untersuchungen zur Theorie der Rhetorik bei Platon und Aristoteles, Gotinga.

Hood, M.D. 1990: Aristotle's Enthymeme: its Theory and Application to discourse, Un. de Oregón 1984. UMI 1990.

Iglesias Zoido, J.C. 1995: La argumentación en los discursos deliberativos de Tucídides y su relación con la normativa retórica del siglo $I V$ a. de C., Universidad de Extremadura.

Kantelhardt, A. 1911: De Aristotelis Rhetoricis, Gotinga.

Kroll, W.: «Rhetorik» en $R E$ cols. 1039 a 1138

Laplace, M. 1995: «Platon et l'art d'écrire des discours: critique de Lysias et d'Isocrate, influence sur Denys d'Halicarnasse», Rhetorica 13, 1-15.

Lavency, M. 1964: Aspects de la logographie judiciaire attique, Lovaina.

Navarre, O. 1900: Essai sur la rhétorique grecque avant Aristote, París.

Nussbaum, M.C. 1996: «Aristotle on Emotions and Rational Persuasion» en A. Oksenberg Rorty, Essays on Aristotle's Rhetoric, Berkeley, pp. 303-323.

Racionero, Q. 1990: Aristóteles. Retórica, Madrid.

Romero Cruz, F. 1987: «En torno a los proemios y epílogos de Tucídides» en G. Morocho Gayo (ed.), Estudios de Drama y Retórica en Grecia y Roma, León, pp. 155-168.

Ryan, E.E. 1984: Aristotle's Theory of Rhetorical Argumentation, Montreal.

Solmsen, F. 1929: Die Entwicklung der aristotelischen Logik und Rhetorik, Berlín.

Schütrumpf, E. 1993: «The model for the concept of ethos in Aristotle's Rhetoric», Philologus 137, 12-17.

Trevett, J.C. 1996: «Aristotle's Knowledge of Athenian Oratory», $C Q$ 46, 371-379.

Todd, S.C. 1994: The Shape of Athenian Law, Oxford.

Vianello de Córdova, P. 1990: «El discurso III de Lisias, Defensa ante Simón: un ensayo hermenéutico», Nova Tellus, 8, 9-60.

Wisse, J. 1989: Ethos and Pathos from Aristotle to Cicero, Amsterdam. 